\title{
ENTRE LA VIRTUD Y EL UTILITARISMO: UNA APROXIMACIÓN A LA EDUCACIÓN MORAL
}

BETWEEN VIRTUE AND UTILITARIANISM:
AN APPROACH TO MORAL EDUCATION

doi: $10.21555 /$ rpp.v33i33.2389

Yaiza Sánchez Pérez

Universidad Internacional de La Rioja, España

yaiza.sanchez@unir.net

https:/ / orcid.org/0000-0002-5895-4355

Recibido: septiembre 1, 2021 - Aceptado: octubre 12, 2021

\section{RESUMEN}

La reflexión sobre cuestiones relacionadas con la educación moral -ya no solo en su concepción teórica, sino en su puesta en práctica dentro de las aulas-, es esencial para el correcto ejercicio de la profesión docente. Por este motivo, se analizan las aportaciones realizadas por Aristóteles y John Stuart Mill a la educación moral, con el fin de construir una reflexión pedagógica que facilite la comprensión del desarrollo moral del alumnado, en el contexto educativo actual. A partir de una perspectiva filosófica, se utiliza un método analítico basado en el estudio de los postulados de la ética de la virtud y el utilitarismo; concretamente en cuestiones relacionadas con el desarrollo moral de los sujetos, la felicidad o la importancia del razonamiento ante los conflictos morales para comprender qué aportaciones pueden aplicarse a la educación actual. Las principales conclusiones extraídas tras el estudio, son la importancia de reflexionar críticamente en los centros educativos sobre conceptos como prudencia, virtud, felicidad o ciudadanía; así como la riqueza que aporta al debate educativo, la incorporación de conceptos como la virtud. Además, desarrollar virtudes en el alumnado permitirá que conozcan el bien, lo deseen y lo practiquen.

Palabras clave: concepto moral, educación, ética, filosofía de la educación, sistema educativo. 


\begin{abstract}
Reflection on issues related to moral education, not only in its theoretical conception, but in its implementation within the classroom, is essential for the right exercise of the teaching profession. For this reason, the contributions made by Aristotle and John Stuart Mill to moral education are analyzed to build a pedagogical reflection that facilitates the understanding of the moral development of students in the current educational context. From a philosophical perspective, an analytical method is used based on the study of the postulates of the ethics of virtue and utilitarianism; specifically on issues related to the moral development of subjects, happiness, or the importance of reasoning in the face of moral conflicts to understand what contributions can be applied to current education. The main conclusions drawn after the study are the importance of critically reflecting in schools on concepts such as prudence, virtue, happiness, or citizenship; as well as the richness that the incorporation of concepts such as virtue brings to the educational debate. In addition, developing virtues in the student body will allow them to know the good, wish it and practice it.
\end{abstract}

Keywords: Education, Ethics, Moral Concepts, Educational Philosophy, Educational System.

\title{
INTRODUCCIÓN Y JUSTIFICACIÓN
}

La educación moral continúa siendo un elemento relevante en la concepción teórica y práctica de la educación. El resurgimiento de modelos como la educación del carácter o la importancia de la educación en valores para la construcción de una ciudadanía democrática, invitan a la comunidad educativa a analizar la moral y la ética en el ámbito educativo y familiar.

El protagonismo adquirido por la individualidad y la competitividad en diferentes ámbitos de nuestra sociedad, ha puesto en cuestionamiento la forma de entender y trabajar los valores dentro del aula. Nos encontramos ante una crisis en la que, de no reflexionar sobre nuestro paradigma educativo, surgirá un sistema en el que se produzcan máquinas utilitaristas y se obvie la función de la educación como una herramienta de construcción de ciudadanos capaces de pensar de manera crítica sobre los acontecimientos, teniendo presentes los logros alcanzados y el sufrimiento ajeno (Nussbaum, 2010).

Con el fin de reflexionar sobre la educación moral en la actualidad, se recurre al análisis de los postulados propuestos por Aristóteles y John Stuart Mill. El objetivo es partir de autores clásicos para comprender qué cabida tienen sus aportaciones en el sistema educativo actual. Al recuperar las contribuciones planteadas por autores clásicos, no se trata de interpretar los planteamientos propuestos por estos autores, sino recuperar los conocimientos que se conocen sobre ellos y aplicarlos a la realidad educativa actual (Kristjánsson, 2015).

El objetivo principal de este estudio es analizar las aportaciones de Aristóteles y John Stuart Mill sobre el desarrollo moral de los sujetos desde una perspectiva pedagógica, concretamente en cuestiones relativas a la felicidad y a la resolución de conflictos morales. De esta manera, se podrá construir una reflexión educativa que facilite la comprensión del desarrollo moral del alumnado en el contexto educativo actual. A nivel metodológico se analizan los planteamientos de Aristóteles y John Stuart Mill desde la ética de la virtud y el utilitarismo. En este sentido, se ha de tener en cuenta que estas perspectivas han sido tradicionalmente consideradas como elementos de investigación, más que una teoría indiscutible con creencias firmes e incuestionables (Jamieson, 2007). Por ello, se utiliza un método analítico, considerando la perspectiva humanística como un elemento imprescindible para 
la construcción de una ciudadanía consciente de sus acciones y con capacidad de replegarse sobre sí misma para generar valores dignos de ser transmitidos.

La temática se aborda, principalmente, a partir de la obra Ética a Nicómaco, de Aristóteles y El utilitarismo, de John Stuart Mill, aunque también habrá argumentos que se apoyen en otras obras representativas como Ética a Eudemo y Sobre la libertad. El análisis se realizará sobre dos cuestiones principales: por un lado, el asunto de la búsqueda de la felicidad como objetivo educativo $\mathrm{y}$, por otro lado, la importancia del razonamiento prudencial para resolver adecuadamente los conflictos morales. El motivo de la elección de estas dos cuestiones se debe a que ambos autores reflexionan explícitamente en sus obras sobre esto y, además, se considera que son dos aspectos de importancia significativa a nivel educativo.

La estructura del estudio se dividirá en tres partes. En la primera, se reflexionará acerca de la búsqueda de la felicidad como objetivo educativo y cómo se ha interpretado este hito desde la ética de la virtud y el utilitarismo. Para abordar esta cuestión, se trabaja el concepto de eudaimonía como principio aristotélico de la felicidad, así como la descripción del «principio de utilidad» utilitarista que defiende la «mayor felicidad para el mayor número». En este primer apartado, también queda reflejado cómo ambas corrientes proyectan la felicidad hacia la comunidad. En la segunda parte, se llevará a cabo una reflexión similar desde ambas perspectivas, pero en este caso, el análisis tratará sobre la resolución de conflictos morales a través del razonamiento. En este apartado se describirá la phronesis aristotélica como virtud y la importancia de la prudencia en la toma de decisiones, además de exponer el planteamiento de «el arte de la vida» de John Stuart Mill para valorar la corrección o incorrección de los actos. En este punto también se incluirán las posibles sanciones que pueden aplicarse a una persona que no predisponga sus conductas hacia la consecución del «principio de utilidad». Por último, se aportan algunas reflexiones finales extraídas tras el estudio, vinculando la investigación con la educación moral en la actualidad.

\section{LA BÚSQUEDA DE LA FELICIDAD COMO OBJETIVO EDUCATIVO}

\section{Aristóteles y el concepto de eudaimonía}

Lograr la felicidad del alumnado es el objetivo educativo por excelencia. Por este motivo, el desarrollo íntegro de los estudiantes es, generalmente, una de las prioridades de los sistemas educativos. Así, es importante comprender qué se entiende por felicidad desde la ética de la virtud y el utilitarismo, además de conocer qué componentes de esta deben tenerse en cuenta para lograr el pleno desarrollo de los alumnos en el contexto educativo actual.

Aristóteles plantea que la felicidad -aquello que él considera el mayor bien- será más perfecta si se procura para la ciudad o la comunidad, por encima del interés individual (Aristóteles, EN, §1094b). Esto refleja la importancia que él otorga al desarrollo cívico y a la educación para la ciudadanía. Por tanto, desde la perspectiva de la ética de la virtud, uno de los elementos principales a tener en cuenta para trabajar la felicidad en el aula, será enfocarla más allá del bienestar y el beneficio individuales; valores como la generosidad, solidaridad y colaboración conformarán una red nomológica que guiará el proceso educativo hacia la felicidad del alumnado.

No obstante, a pesar de que la felicidad sea un bien por excelencia, necesita de bienes exteriores a ella, ya que es importante que existan unas condiciones óptimas para ser feliz. Esto es lo que Aristóteles denomina la buena suerte, es decir, que la felicidad necesita de prosperidad (Aristóteles, EN, §§ 1099b-1100a). De este modo, se entiende que lograr la felicidad no 
siempre depende de uno mismo, sino que tener los recursos adecuados y las circunstancias favorables, ayuda notablemente a alcanzar la felicidad. Desde esta perspectiva, tener buena suerte es algo irracional y la única explicación posible, según Aristóteles, es la voluntad de Dios (Aristóteles, EE, §1248a). No obstante, a pesar de las dificultades, la felicidad puede ser alcanzada por el aprendizaje y el ejercicio (Aristóteles, EN, § 1099b). Por este motivo es fundamental que en el aula se muestren las circunstancias reales que pueden encontrarse al buscar la felicidad, unas más favorables que otras. Sin embargo, resulta imprescindible hacer hincapié en la necesidad de desarrollar hábitos de pensamiento y acción para lograr la vir$t u d$, independientemente de las circunstancias que al alumno le haya tocado vivir. La virtud aristotélica es definida como la disposición hacia la excelencia (Aristóteles, EN, § 1105b), conductora hacia la felicidad como fin último (Annas, 1998), aunque necesita de un equilibrio con el resto de virtudes para que la felicidad sea plena. La buena o mala suerte es algo independiente de la persona; por tanto, hay que insistir en la labor que depende de uno mismo y plantearse cómo lograr la felicidad a pesar de las dificultades.

Aristóteles, en su obra Ética a Nicómaco, especifica que la felicidad ha de tener un valor intrínseco, debe ser autosuficiente y que nada le haga aumentar su valor (Aristóteles, EN, § 1097b). Esta también ha de ser activa con el fin de vivir y hacer el bien; debe estar disponible para todas aquellas personas que actúen para alcanzarla, pues la felicidad no puede verse limitada al disfrute de unos pocos: debe ser relativamente estable y que no se esfume cuando ocurra algo desafortunado (Nussbaum, 2004; West, 1982).

El estagirita relaciona la felicidad con el bien más perfecto, ya que «se elige por sí mismo y nunca por otra cosa» (Aristóteles, EN, § 1097a, p. 140). Concretamente, vincula la felicidad con el concepto de eudaimonía (gr. $\varepsilon v \delta \alpha \mu \nu$ ovı $\alpha$ ) (Aristóteles, EN, § 1097b). La eudaimonía puede definirse como la felicidad que es posible alcanzar mediante el uso de la razón, incluyendo actividades valiosas de tipo ético, intelectual o político, vinculadas a una virtud perfecta (Aristóteles, EN, § 1099b). En esta definición, también se incluyen actividades relacionadas con el amor y la amistad (Nussbaum, 2004), mostrando así la importancia de las relaciones interpersonales y de las emociones para el correcto desarrollo de la virtud.

A partir de las propuestas de Aristóteles, se puede deducir la importancia de algunos elementos imprescindibles para lograr la felicidad como virtud y trabajarla en el aula: por un lado, que se proyecte para toda la comunidad por encima del interés individual; por otro, la posible influencia de la buena suerte moral para alcanzar la felicidad, aunque hay que tener en cuenta que esto puede ser necesario, pero no suficiente, para lograr la felicidad y, por último, la necesidad de buscar la felicidad a partir de la razón, las relaciones interpersonales y las emociones. Potenciar estos aspectos en los centros educativos es necesario para que el alumnado desarrolle todas sus dimensiones humanas de manera equilibrada.

La ética de la virtud ha de centrarse en la acción y, para ello, es importante que en la escuela se creen espacios para pensar, sentir y actuar conforme a la virtud y a la búsqueda de comportamientos moralmente buenos. Trabajar la búsqueda de la felicidad en las aulas reflejando la importancia de la comunicación, las relaciones interpersonales y la convivencia en sociedad, permite al alumnado ser consciente de la necesidad de la colaboración con el otro para lograr ser buena persona y buen ciudadano.

\section{John Stuart Mill y el «principio de utilidad»}

El utilitarismo, por su parte, fue fundado por Jeremy Bentham y John Stuart Mill durante el siglo XVIII. Ambos autores comparten unas líneas generales, aunque con ciertos matices: coinciden en la necesidad de promover la mayor felicidad al mayor número de personas 
como único bien, de manera que las acciones deseables son las que promueven la felicidad, y las indeseables, las que disminuyen la misma.

John Stuart Mill estuvo influenciado por su padre, James Mill, a la hora de configurar su visión educativa (Finlay, 2017; Mill, 2009; Viner, 1949; Xiem, 2018). Uno de los puntos débiles de la concepción educativa de Mill era la educación emocional, puesto que su padre le había inculcado la importancia de destacar a nivel intelectual, pero le transmitió un sentimiento de vergüenza ante determinadas emociones (Fuentes Jiménez, 2010; Nussbaum, 2004). No obstante, parece desvincularse de esas influencias a partir de 1828, siendo su discurso Perfectibility el punto de inflexión. En este discurso, se observa un progreso en sus propias ideas. John Stuart Mill pasó a considerar la educación desde una perspectiva holística, incorporando la dimensión intelectual, y la necesidad de considerar la dimensión emocional para lograr un proceso educativo óptimo y equilibrado. En este discurso, Mill interpreta que «las pasiones son la primavera, el principio moral es solo el regulador de la vida humana» (Mill, 1828). Se refuerza así la importancia de la dimensión emocional en el desarrollo moral de las personas.

Otro de los aspectos que Mill destaca en su obra es que las acciones son decisivas y predominantes para conseguir la felicidad (Mill, 2014a). En este sentido, el autor vincula directamente la felicidad con la utilidad y la convierte en principio fundamental de sus aportaciones, llegando a considerar la felicidad como placer y ausencia de dolor.

El criterio para que una acción sea considerada utilitarista no es que aporte una felicidad individual, sino más bien de que haya una «mayor cantidad de felicidad total» (Mill, 2014a, p. 69). Al igual que ocurría con el planteamiento aristotélico, predomina la preferencia de la felicidad comunitaria por encima del bienestar individual. En esta línea, Mill llega a plantear la posibilidad de que una persona sacrifique su propia felicidad. Según el autor, el verdadero objeto de la virtud es multiplicar la felicidad (Mill, 2014a). No obstante, advierte que esta acción debe tener un objetivo: renunciar a la felicidad propia en favor de la comunidad. En caso de que la renuncia a la felicidad personal se produzca para obtener otro tipo de beneficios, no merece respeto ni admiración. Sobre esto, Mill apunta: «Un sacrificio que no incremente o tienda a incrementar la suma total de la felicidad, se considera como inútil» (Mill, 2014a, p. 79). El planteamiento del autor es claro en cuanto a que, si el mayor número de personas no logra la felicidad, es digno de alabar que, para alcanzarla, alguien renuncie conscientemente a su felicidad o a la posibilidad personal de alcanzarla.

Para cumplir con el principio utilitarista de la mayor felicidad para el mayor número, Mill trata de equiparar el valor del razonamiento práctico y el teórico (Kreider, 2010; West, 1982). Lo que confirma que la percepción de Mill sobre la felicidad puede comprenderse gracias a las influencias recibidas tanto de Bentham como de autores clásicos, como Aristóteles (Nussbaum, 2004).

En el aula es posible transmitir esta visión de la felicidad basada en la entrega y la generosidad de manera desinteresada. Sin embargo, es importante mostrar las consecuencias que tiene el hecho de sacrificar la propia felicidad en favor de los demás. Es decir, comprender dónde enfoca su felicidad: en sí mismo o en el bienestar de las personas más próximas.

Con todo, es posible encontrar algunos criterios diferenciadores entre la propuesta aristotélica y la propuesta de Mill. En el caso del utilitarismo, el fin de la felicidad es una existencia libre de dolor y llena de placer, tanto en calidad como en cantidad (Mill, 2014a). Por su parte, Aristóteles vincula la felicidad al bien más perfecto y lo relaciona con el concepto de eudaimonía, un elemento en constante desarrollo y necesario para el perfeccionamiento de las propias virtudes (Kristjánsson, 2015), lo que convierte a la felicidad en un fin en sí mismo. Del mismo modo que Aristóteles atribuía la infelicidad, entre otras causas, a la falta de buena 
suerte moral, John Stuart Mill considera que el planteamiento utilitarista no solo se basa en la búsqueda de la felicidad, sino que, además, previene y alivia la infelicidad (Mill, 2014a). En ningún caso debe confundirse e interpretarse la felicidad como un estado placentero prolongado en el tiempo, ya que esto resultaría una quimera.

No obstante, también se pueden apreciar algunas similitudes. Ambos son partidarios de la eudaimonía y lo que esta aporta a la noción de felicidad, tal y como ellos la conciben. Además, los dos autores aspiran a lograr una maximización de la felicidad en el ámbito público (Gozálvez y Jover, 2016) con el fin de favorecer el bien común.

\section{LA IMPORTANCIA DEL RAZONAMIENTO EN LOS CONFLICTOS MORALES}

\section{La phronesis aristotélica como ejercicio prudencial}

El planteamiento principal de la ética aristotélica es que el centro del proceso educativo ha de ser la acción, la realización de comportamientos moralmente buenos (Aristóteles, EN, $\S 1094 a)$. Desde esta perspectiva, actuar correctamente es fundamental para lograr ser una persona virtuosa. El comportamiento moral, por tanto, se adquiere al interiorizar determinadas virtudes que son transmitidas y que se han puesto en práctica de manera prudencial, no mecánica (Fuentes, 2018). Las virtudes morales surgirán del ejercicio guiado por la prudencia (García Amilburu y García Gutiérrez, 2012), y su consecuencia más directa será la transformación y la mejora del ser humano.

La prudencia -una de las virtudes dianoéticas clasificadas por Aristóteles (Aristóteles, EN, $\S 1102 \mathrm{~b}$ ) - es una de las virtudes más importantes propuestas por el estagirita, considerada incluso la virtud más perfecta (Bernal, 1998), ya que a partir de ella pueden desarrollarse el resto de virtudes (Berkowitz, 1995). Aristóteles define la prudencia como «un modo de ser racional verdadero y práctico, respecto de lo que es bueno y malo para el hombre» (Aristóteles, EN, § 1140b). Puede considerarse la prudencia como una virtud imperativa, puesto que la persona prudente acepta llevar a cabo una acción (Zagal, 2012) tras un ejercicio de reflexión, convirtiendo las acciones en decisiones deliberadas (Vigo, 2012). La prudencia es la virtud que permite a la persona analizar una situación particular para decidir, tras un proceso deliberativo, qué es lo más adecuado en cada momento, evitando así actuar con impulsividad ante cualquier estímulo.

El razonamiento prudencial o sabiduría práctica, también denominada phronesis, se adquiere mediante un proceso de deliberación previo y la interiorización de determinados hábitos hasta convertirlos en una forma de vida. De esta manera, desde el planteamiento aristotélico, no se trata tanto de conocer qué es el bien, sino también de actuar moralmente bien (Bernal et al., 2015). Es igual de importante conocer qué es el bien como practicarlo. Tal y como la define Kristjánsson (2015): «La phronesis es el dominio armonioso de toda la vida» (p. 97). Es necesario puntualizar que la sabiduría práctica no busca verdades absolutas como lo puede hacer la razón, sino que busca el mejor resultado posible para lograr el florecimiento humano (Carr, 2016). De este modo se logra un equilibrio entre lo cognitivo y lo comportamental. No obstante, no hay que olvidar la importancia que adquieren las emociones en el desarrollo moral del alumnado.

Desde la ética aristotélica, cuando dos virtudes entran en conflicto, se propone el ejercicio de la razón y el autodominio, destacando la importancia del desarrollo de virtudes 
que permitan a la persona actuar en el sentido correcto (García Amilburu y García Gutiérrez, 2012). La deliberación práctica o phronesis es útil para discernir entre las diferentes razones para actuar en una situación particular, por lo que es importante destacar que no es susceptible de codificación o clasificación en reglas generales (Carr, 2016). En este sentido, se plantea que la deliberación sea la herramienta que permita a la persona extraer conclusiones sobre los actos y sus consecuencias. Por tanto, la phronesis es considerada como una virtud integradora (Kristjánsson, 2015) al analizar cada situación particular y valorar cómo y por qué actuar de una determinada forma, cuando dos valores entran en conflicto. El objetivo es que, al desarrollar la virtud de la prudencia, se logren todas las demás, tal y como indicaba Aristóteles. En palabras de Schwartz y Sharpe (2010):

Practical wisdom is a kind of moral jazz. It sometimes depends on rules and principles -like the notes on the page and the basic melodies in jazz. But rules by themselves can't do the job. Moral improvisation is the interpretative tune we play around these notes and melodies, in order to do the right $\operatorname{thing}^{1}$ (p. 41).

Es necesario tener presente cuáles son las virtudes y las reglas morales más favorables socialmente, pero también es fundamental el proceso deliberativo sobre cada situación particular, en el que se decida qué pensamientos, voluntades y comportamientos han de priorizarse cuando dos virtudes entran en conflicto, independientemente de qué regla moral deba de ser aplicada.

Además de todos los beneficios que aporta la sabiduría práctica a la formación de la integridad del ser humano, es importante señalar que el desarrollo del razonamiento prudencial o phronesis no solo beneficia al crecimiento de la persona en tanto que se produce una mejora como ser humano, sino que el aprendizaje de este proceso deliberativo frente a una toma de decisiones, favorece igualmente a su formación profesional: todos los aprendizajes técnicos y específicos, deben ir acompañados de una reflexión deliberativa y prudencial (Kristjánsson, 2015). Por tanto, la prudencia como virtud, no debe ser una cuestión ajena a los planes de estudio, independientemente de que un sistema educativo ponga el foco en la profesionalización del alumnado y la priorice sobre su desarrollo personal.

Adquirir la virtud de la prudencia permitirá que el alumnado sea reflexivo, desarrolle la capacidad para afrontar dificultades y adquiera una destreza para resolver problemas en la vida cotidiana, tratando de encontrar la mejor solución en cada caso y afrontando posibles conflictos morales. Por ello, es fundamental que en los centros educativos se generen espacios físicos y temporales para realizar ejercicios prudenciales, con el fin de que los alumnos tomen conciencia de qué es lo mejor, por qué lo desean y cómo pueden actuar para lograrlo.

\section{El «arte de la vida» de John Stuart Mill en la evaluación de las conductas morales}

Tomando como referencia la propuesta utilitarista de Mill, que promocionar la felicidad sea deseable, no significa que sea obligatoria ${ }^{2}$, Mill expuso que la obligación moral supone el

1 La sabiduría práctica es una especie de jazz moral. A veces depende de reglas y principios, como las notas de la página y las melodías básicas del jazz. Pero las reglas por sí solas no pueden hacer el trabajo. La improvisación moral es la melodía interpretativa que tocamos en torno a estas notas y melodías para hacer lo correcto. (Traducción).

2 Es necesario distinguir entre las acciones valiosas y obligatorias (Nakhnikian, 1951). Mill identifica las acciones valiosas con el placer, estableciendo una relación entre lo bueno y lo deseable; pero ello no implica que haya una correlación directa entre lo bueno y lo deseado; además, las acciones obligatorias se basan en la relación de esas acciones valiosas con sentimientos éticos. 
cumplimiento de las acciones que contribuyen a mejorar la sociedad (Mill, 2014a), por tanto, hay que asumir que existen acciones concretas para desarrollar los principios moralmente obligatorios, pero esto implica un costo: enseñar y aprender dichas acciones ${ }^{3}$.

Teniendo en cuenta este planteamiento, se ha llegado a interpretar la aportación de Mill desde una perspectiva en la que las obligaciones morales son mínimas (porque los beneficios de establecer una norma moral son menores que sus costos), y no se busca una maximización de la regla de la utilidad. Con el objetivo de recoger todos estos matices, Edwards (1986) propone una interpretación del primer «principio de moralidad» de Mill, incluyendo cuatro formas del deber y teniendo en cuenta los aspectos de justicia y obligación moral: 1) abstenernos de infligir daño; 2) prevenir activamente el daño; 3) proporcionar y proteger activamente elementos mínimos de cualquier tipo de bienestar, y 4) realizar aportes ocasionales, limitados e inespecíficos a la caridad, que aún no llegan a maximizar el bienestar de nadie.

Ante este escenario, es común que los seres humanos se encuentren ante una situación en la que dos actos se deban hacer porque la moralidad así lo requiere. En una circunstancia así, es posible que se inicie un conflicto interno que derive en sentimientos de frustración o infelicidad. De esta manera, se produce un enfrentamiento de valores, lo que muestra que el planteamiento utilitarista que busca la maximización de la felicidad puede reflejar ciertas desventajas (Gaus, 1980). La aparición de un posible conflicto de intereses es el único escenario en el que Mill podría llegar a calificar directamente las acciones como correctas e incorrectas, puesto que los utilitaristas «son perfectamente conscientes de que existen otras posesiones y cualidades deseables aparte de la virtud [...] y también son conscientes de que una acción correcta no indica generalmente una persona virtuosa» (Mill, 2014a, pp. 85-86). Siendo más específicos, es una manera de describir las obligaciones opuestas de manera que no se puntualice explícitamente la utilidad de cada acción alternativa en una situación particular (Lyons, 1976). En un caso como este en el que se haga frente a dos obligaciones morales opuestas, se debería mostrar una posición favorable hacia la regla que menos viole el «principio de utilidad» (Edwards, 1985).

A pesar de mostrar una clara predisposición de preferir la opción que menos quebrante el "principio de utilidad», las conductas humanas no siempre cumplen con esta formulación y, en ese caso -según Mill-, deberán ser sancionadas. La sanción definitiva de una norma moral es la propia conciencia, es decir, el sentimiento de culpa o remordimiento (Mill, 2014a). Sin embargo, hay sujetos que carecen de conciencia, por lo que es preciso que existan sanciones externas, como la aplicación de la legalidad vigente (Jacobson, 2008; Nakhnikian, 1951; Riley, 2017) o la desaprobación general (Mill, 2014b). Tal y como indica el propio Mill: «[...] Las recompensas y castigos externos, ya sean físicos o morales, ya procedan de Dios o de nuestros semejantes [...] pueden ser utilizados para reforzar la moralidad utilitarista» (Mill, 2014a, p. 99). Esto lleva a cuestionar si los sentimientos de conciencia son innatos o implantados por agentes externos, propuesta por la que se decanta el autor al considerar que son claramente adquiridos. En su obra Sobre la libertad apunta:

Decir que los deseos y sentimientos de una persona son más fuertes y más diversos que los de otra, es decir simplemente que tiene más materia prima de naturaleza humana y que, por tanto, es capaz quizá de hacer un mayor mal, pero seguramente también de hacer un mayor bien (Mill, 2014b, p. 73).

3 Estas pautas de actuación se consideran morales cuando previenen algún mal o proporcionan un beneficio mayor que el costo que ellas implican, por lo que no buscan maximizar el bien de la humanidad, sino minimizar el mayor daño posible (Edwards, 1985, 1986; Lyons, 1976). 
El autor considera que la educación y las sanciones externas pueden utilizarse para que los sujetos creen asociaciones de ideas y generen otros sentimientos. En definitiva, de esta manera se genera un sentimiento natural en el que la utilidad implica hacer lo correcto, algo que remite al concepto de justicia (Mill, 2014a), la regla moral por excelencia en el pensamiento de John Stuart Mill.

La visión teleológica del utilitarismo de Mill es también llamada «arte de la vida»y se compone por tres elementos: moralidad, estética y prudencia, que determinan qué es lo digno y lo deseable (Mill, 2014a). Estos tres aspectos son los que permiten evaluar la corrección o incorrección de los actos. La diferencia entre ellos es la siguiente: las reglas morales son órdenes categóricas, respaldadas por sanciones (externas e internas); la estética está vinculada a lo correcto moralmente, lo conveniente y lo hermoso en lo que respecta a la conducta humana y, por último, las acciones prudenciales se basan en enunciados hipotéticos (Ryan, 1970), por ejemplo, si no quieres caerte, no te acerques al precipicio.

Una de las principales preocupaciones de Mill era la lógica del razonamiento prudencial y su relación con el razonamiento moral, por lo que tomó como referencia un hedonismo universal (Mill, 2014a) con el objetivo de desarrollar un razonamiento práctico que incluyera ambas perspectivas (Brown, 1973). Desde este planteamiento, no se evalúan únicamente las reglas morales, pues la moralidad solo es una parte de la evaluación que realiza el «principio de utilidad» (Gaus, 1980), sino que además se busca alcanzar la mayor cantidad total de felicidad por encima de la felicidad y el placer individual (Mill, 2014a). La utilidad mantiene así un compromiso con la felicidad como fin único deseable, valorando que todos los elementos puedan servir como medio para alcanzarla (Gaus, 1980; Lyons, 1976) y, a ser posible, que sus efectos se expandan hacia el mayor número de personas posible. La utilidad se convierte así en un principio evaluador de la conducta humana, independientemente de que dicha evaluación se aplique desde la moralidad, la prudencia o la estética.

Todas estas aportaciones sitúan siempre el foco en el sujeto como agente activo del razonamiento y la acción moral, lo que refleja una clara posición individualista y liberal del pensamiento de John Stuart Mill. Esto aporta información sobre cómo se configura una visión individualista de la construcción del carácter, entendiendo al sujeto como principal generador y receptor de las propias acciones morales, independientemente de que estas tengan repercusión en la comunidad.

Educar moralmente al alumnado desde una perspectiva utilitarista, implica enseñarles que hacer lo correcto es hacer lo útil y esto, a su vez, se traduce en lograr la felicidad para el mayor número de personas. Desde este planteamiento, obrar correctamente no te convierte en una persona virtuosa, simplemente no consideran prioritario el desarrollo de la virtud porque, a pesar de considerarlo necesario, entienden que no garantiza el cumplimiento del «principio de utilidad». La valoración de las conductas humanas a partir de los componentes del «arte de la vida» (moralidad, estética y prudencia) es una herramienta más para buscar la mayor felicidad para el mayor número. En caso de no lograr el cumplimiento del «principio de utilidad», las personas se enfrentarán a sanciones, siendo su propia conciencia la mayor de las sanciones al sentir que no hacen «lo correcto» y no contribuyen a lograr la mayor cantidad total de felicidad.

Todo ello nos hace reflexionar sobre las aportaciones de John Stuart Mill a la educación actual: por un lado, la defensa de la justicia y la utilidad en las conductas, como elementos centrales de su propuesta; el uso de la razón para determinar cuál 
es la mejor alternativa, en caso de que dos cuestiones morales entren en conflicto; la influencia emocional en la configuración moral de la persona; la presencia de reglas morales generales que permitan evaluar el comportamiento deseable de los sujetos o las posibles sanciones que puede enfrentar la persona, en caso de no favorecer el logro de la mayor felicidad para el mayor número.

\section{ALGUNAS CONSIDERACIONES FINALES}

Este análisis ha permitido que, a partir de los planteamientos expuestos por autores clásicos, se muestren dos caminos diferentes para interpretar la búsqueda de la felicidad y la resolución de conflictos morales en el contexto educativo actual. Tras el análisis realizado, se proponen algunas reflexiones finales.

Por un lado, conceptos aristotélicos como la eudaimonía han estado presentes en planteamientos posteriores sobre educación moral. Tal es así, que este término fue adoptado por John Stuart Mill para la configuración de su teoría y aún hoy tiene una gran influencia en enfoques vigentes como la educación del carácter. La educación del carácter, durante los últimos años, ha tomado influencias de diferentes corrientes (Bernal et al., 2015) y autores (Kohlberg, 1992; Peterson y Seligman, 2002) que le han permitido amoldarse a las necesidades que han ido surgiendo como consecuencia del dinamismo social. Desde este enfoque, la eudaimonía se considera una actividad permanente que necesita el desarrollo pleno de las capacidades humanas para tomar decisiones conforme a la virtud (Fowers, 2012). En este sentido, la educación del carácter plantea la existencia de tres dimensiones a desarrollar en el ser humano: una dimensión cognitiva, otra afectiva y otra conductual (Williams, 2000), todas ellas imprescindibles e interdependientes. Esta triangulación de conceptos (pensamiento, sentimiento y comportamiento) muestra una extraordinaria consonancia con la concepción de Mill sobre el «arte de la vida», entendida como el equilibrio entre la moralidad, la prudencia y la estética para valorar los comportamientos morales. Además, el enfoque de la educación del carácter, de origen aristotélico, permite que el alumnado conozca qué es el bien, lo desee y lo practique (Lickona, 1991). Esto demuestra la relación directa que existe entre los planteamientos clásicos con los enfoques actuales de educación moral. Por tanto, es necesario continuar reflexionando sobre estos conceptos con el fin de intervenir, evaluar y mejorar su implementación en los centros educativos.

Por otro lado, es fundamental reflexionar críticamente en las aulas sobre conceptos como la felicidad, el placer, la prudencia o la conducta moral. El desarrollo del pensamiento crítico resulta esencial, aunque actualmente continúa siendo una competencia que, en la práctica, resulta compleja para algunos docentes por la falta de recursos para su desarrollo. Por este motivo, se considera esencial que, en las aulas, los alumnos no solo identifiquen qué es el bien, sino que lo practiquen, de tal manera que comiencen a adquirir conciencia de sus obligaciones morales y las consecuencias que estas poseen a nivel individual y social.

Por último, resulta enriquecedor recuperar un concepto como la virtud en el debate educativo actual. Es un concepto que, tanto Aristóteles como Mill, mencionan en sus planteamientos como objetivo a desarrollar, aunque el primero lo considera como algo ineludible y, el segundo, un aspecto necesario, pero no suficiente. En este sentido, si la educación moral se traduce únicamente en la enseñanza de valores, esta última quedaría relegada a un plano intelectual, puesto que solamente permite al alumnado reconocer, desde una dimensión cognitiva, cuáles son los valores socialmente aceptados; mientras que el desarrollo de virtudes 
va más allá y se enfoca a la acción y al comportamiento, a la deliberación e interiorización de hábitos que produzcan un cambio en la persona. Al fin y al cabo, el objetivo de toda acción educativa ha de ser la transformación y mejora del ser humano.

\section{REFERENCIAS}

Annas, J. (1998). Virtue and Eudaimonism. Social Philosophy E Policy Foundation, 37-55.

Aristóteles (2019). Ética a Nicómaco y Ética a Eudemo. Gredos.

Berkowitz, M. W. (1995). Educar la persona moral en su totalidad. Revista Iberoamericana de Educación, 8, 73-101.

Bernal, A. (1998). Educación del carácter, educación moral: propuestas educativas de Aristóteles y Rousseau. EUNSA.

Bernal, A., González-Torres, M. C. y Naval, C. (2015). La educación del carácter. Perspectivas internacionales. Participación educativa, 35-45.

Brown, D. G. (1973). What is Mill's Principle of Utility? Canadian Journal Of Philosophy, III(1), 1-12.

Carr, D. (2016). Virtue and Knowledge. Philosophy, 91, 375-390. https:/ / doi.org/10.1017/ $\underline{\text { S003181911600005X }}$

Edwards, R. B. (1985). J. S. Mill and Robert Veatch's Critique of Utilitarianism. The Southern Journal of Philosophy, 23(2), 181-200.

Edwards, R. B. (1986). The Principle of Utility and Mill's Minimizing Utilitarianism. The Journal of Value Inquiry, 20, 125-136.

Finlay, G. (2017). Mill on Education and Schooling. In A Companion to Mill (Eds. Christopher Macleod y Dale E. Miller). John Wiley \& Sons, Inc.

Fowers, B. J. (2012). An Aristotelian Framework for the Human Good. Journal of Theoretical and Philosophical Psychology, 32(1), 10-23. https:/ / doi.org/10.1037/a0025820

Fuentes Jiménez, J. R. (2010). Educación para la libertad en Stuart Mill. Revista Miscelánea de Investigación, 23, 139-164.

García Amilburu, M. y García Gutiérrez, J. (2012). Filosofía de la educación. Cuestiones de hoy $y$ de siempre. Narcea y UNED.

Gaus, G. F. (1980). Mill's Theory Of Moral Rules. Australasian Journal of Philosophy, 58(3), 265-279.

Gozálvez, V. y Jover, G. (2016). Articulación de la justicia y el cuidado en la educación moral: Del universalismo sustitutivo a una ética situada de los derechos humanos. Educación XX1, 19(1), Article 1. https:/ / doi.org/10.5944/educxx1.15588

Jacobson, D. (2008). Utilitarianism without Consequentialism: The Case of John Stuart Mill. Philosophical Review, 117(2), 159-192.

Jamieson, D. (2007). When Utilitarians Should Be Virtue Theorists. Utilitas, 19(2), 160-183.

Kohlberg, L. (1992). Psicología del desarrollo moral. Desclée de Brouwer. 
Kreider, S. E. (2010). Mill on Happiness. Philosophical paper, 39(1), 53-68.

Kristjánsson, K. (2015). Aristotelian Character Education. Routledge.

Lickona, T. (1991). Educating for character: How our schools can teach respect and responsibility. Bantam.

Lyons, D. (1976). Mill's Theory of Morality. Noûs, 10(2), 101-120.

Mill, J. S. (1828). Speech on Perfectibility. https:// www.utilitarian.org/texts/perfectibility. $\underline{\mathrm{html}}$

Mill, J. S. (2009). Autobiography of John Stuart Mill. The Floating Press.

Mill, J. S. (2014a). El utilitarismo. Un sistema de la lógica (Libro VI, capítulo XII). Alianza Editorial.

Mill, J. S. (2014b). Sobre la libertad. Akal.

Nakhnikian, G. (1951). Value and Obligation in Mill. Ethics.

Nussbaum, M. (2004). Mill between Aristotle \& Bentham. Daedalus, 133(2), 60-68.

Nussbaum, M. (2010). Sin fines de lucro. Por qué la democracia necesita de las humanidades. Katz editores.

Peterson, C. \& Seligman, M. E. P. (2002). The values in action (VIA) classification of strengths. A life worth living: Contributions to positive psychology, 29-48.

Riley, J. (2017). Mill on Utilitarian Sanctions. In Christopher Macleod \& Dale E. Miller (Eds.). A Companion to Mill. John Wiley \& Sons, Inc.

Ryan, A. (1970). The Philosophy of John Stuart Mill. MacMillan.

Schwartz, B. \& Sharpe, K. (2010). Practical Wisdom: The Right Way to Do the Right Thing. Penguin.

Vigo, A. G. (2012). Deliberación y decisión según Aristóteles. Tópicos, 43, 51-92.

Viner, J. (1949). Bentham and J. S. Mill: The Utilitarian Background. The American Economic Review, 39(2), 360-382.

West, H. R. (1982). Mill's «Proof» of Utility. In Miller \& Williams (Eds.), The Limits of Utilitarianism. University of Minessota Press.

Williams, M. M. (2000). Models of Character Education: Perspectives and Developmental Issues. Journal Of Humanistic Counseling, Education And Development, 39, 32-40.

Xiem, N. T. (2018). John Stuart Mill's Liberal thought on Education and the Dissemination of Education in Enforcing the Right of Liberty. American Journal of Educational Research, 6(5), 570-577.

Zagal, H. (2012). Apetito recto, prudencia y verdad práctica Las pautas de la eupraxía en la Nicomáquea. Metafísica y persona. Filosofía, conocimiento y vida, 9, 91-111. 